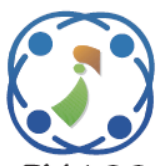

\title{
Marathi Numeral Identification System in Devanagari Script Using 1D Discrete Cosine Transform
}

\author{
Madhav Vaidya $^{{ }^{*}} \quad$ Yashwant Joshi $^{1} \quad$ Milind Bhalerao $^{1}$ \\ ${ }^{1}$ Shri Guru Gobind Singhji Institute of Engineering and Technology, Vishnupuri, Nanded, India \\ * Corresponding author's Email: mvvaidya@gmail.com
}

\begin{abstract}
Recognition of handwritten Marathi character/digits is comparatively a tough task as compared to English. It has several types of applications including the postal code reading and sorting, banks check amount processing. In this paper a novel feature extraction and selection method is proposed for the recognition of isolated handwritten Marathi numbers which is based on one dimensional Discrete Cosine Transform (DCT) algorithm. The scanned document is pre-processed and segmented to create isolated numerals. Features for each numeral can be calculated after normalizing the numeral image to $32 \times 32$ size. Based on these reduced features, the numerals are classified into appropriate groups. Neural network is used for classification of numerals based on the extracted features. The results of proposed method are compared with the results obtained using Discrete Wavelet Transform and zonal discrete cosine transform (ZDCT). The proposed approach gives improved results as compared to zonal DCT and DWT method. Experimental results shows accuracy observed for the method is $90.30 \%$ with normalized numeral image of size $32 \times 32$.
\end{abstract}

Keywords: Devanagari, Marathi numeral, OCR, DCT, DWT, Feature extraction.

\section{Introduction}

Optical Character Recognition (OCR) systems primarily convert the scanned document into editable text, audio or speech format which can be stored in digital format. OCR converts scanned text/character images into editable text format. In this paper focus is given on Marathi numeral recognition. Marathi language uses Devanagari script for writing. It is mainly spoken by most of the peoples from Maharashtra state. As Marathi is using decimal numbering system, there are ten distinct numerals from 0 to 9 . The structure of Marathi Numerals is different from English number system. Thus the structural features obtained are different as compared to English, but there are some numerals which seems similar in handwritten format though it is having some differentiation in printed form. Table 1 shows the numeral representation of numbers from 0 to 9 in Marathi language. Basically recognition systems are developed for either online or offline character or numeral recognition. Most of the researchers worked on offline OCR systems. In this paper we have considered offline numeral database for experimentation.

Table 1. Marathi numerals representation from 0 through

\begin{tabular}{|c|c|c|c|c|c|}
\hline Numeral & $\begin{array}{l}\text { Marathi } \\
\text { Number }\end{array}$ & $\begin{array}{c}\text { Trans- } \\
\text { literation }\end{array}$ & Numeral & $\begin{array}{l}\text { Marathi } \\
\text { Number }\end{array}$ & $\begin{array}{c}\text { Trans- } \\
\text { literation }\end{array}$ \\
\hline 0 & शून्य & śūnya & 4 & पाच & Pāch \\
\hline$?$ & एक & ek & $\xi$ & सहा & Saha \\
\hline २ & दोन & don & $७$ & सात & sāt \\
\hline ३ & तीन & tīn & $<$ & आठ & àth \\
\hline$\gamma$ & चार & chār & $\rho$ & नऊ & nau \\
\hline
\end{tabular}


DCT is used for feature space reduction. First summation vectors of normalized numeral image are calculated in horizontal, vertical and diagonal direction (projection profile vectors) PFV. These vectors are concatenated. Also Histogram of gradients HOG is calculated for the same numeral. DCT is separately applied on these two vectors PFV and HOG. First 25 percent coefficients are selected to form each vector to form a new feature vector containing less number of dimensions.

The remaining paper is organized as follows. Section 2 describes the related work where DCT is used in research for different purposes. Section 3 describes the detailed method for recognition of Marathi Numerals which consist of image acquisition, pre-processing, segmentation and size normalization and the proposed approach for feature selection using 1D DCT. Experimental results along with comparisons with existing methods are discussed in Sect. 4. In the last Sect. 5, we conclude the work.

\section{Related work}

Main OCR research application is aiming to reduce human efforts and produce error-free system running in post office work [1], also to reduce the work in different areas like, automation systems for reading numerals [2]. As India is having different scripts in different regions, recognition methods studied so far are discussed by $\mathrm{U} \mathrm{Pal}[3,4]$.

Niranjan Das used topological features for classification [5], likewise statistical features are also used for feature extraction [6-7]. Combination of horizontal and vertical summation vector is used to form feature vector. Work is also carried out for Numeral recognition in many scripts like Bangla [8], Oriya [9] and Kannada [10]. The first most important issue or step in recognition process is the separation of character using segmentation. In this stage of implementation any error or inaccuracy will affect the overall accuracy of the approach used for recognition.

Among all the emerging standards for image and video compression are JPEG (Joint Photography Expert Group), for compression of still images [11], MPEG (Motion Picture Expert Group), for compression of motion video. All above mentioned standards employ a basic technique known as the Discrete Cosine Transform (DCT) [12]. The mathematical formulation by Ahmed is used in most of the literature. DCT is a closely equivalent to the Discrete Fourier Transform (DFT) which converts a given signal or an image from the spatial domain to the frequency domain. The DCT is a transform which converts a signal into elementary frequency components. Every discrete cosine transform uses $\mathrm{N}$ orthogonal real basis vectors having components as cosines. The excellent energy compaction property of DCT which requires only real operations in transformation process is very important property of DCT.

In most of the comparison with the Template methods, the normalization of the position and the size of the feature is carried out for comparing the features for matching purpose $[13,14]$ and the performance of the normalization is very important in order to get remedy from the situation for sampling in advance. Fuzzy logic also used for preprocessing and segmentation in some research [15]. Most of the clustering and classification algorithms used for recognition are elaborated in [16]. Segmentation based approach was proposed by Shaw [17]. Template matching is also very straight forward approach for recognition which is used for recognition whole word or string of digits using holistic approach [18]. Fuzzy logic also can be used for recognition purpose [19]. Park J worked on whole word recognition without segmenting it [20]. Document Text retrieval without using optical character recognition also can be performed [21] along with the minimization in the error rate [22]. Some shapes can be used [23] and verification of recognized result can be performed for enhancing the accuracy [24].

DCT is popular transform used in most of the face recognition applications for extracting local and global features [25, 26]. 1D DCT is used and applied on horizontal and vertical projection profiles to reduce the dimensions [27]. On the basis of the above observations, we were motivated by the need to develop progressive matching approach on the basis of the DCT for the recognition of handwritten Marathi digits.

In our approach feature vector is obtained by using projection profiles and Histogram of Gradients for normalized numeral binary image of size $32 \times 32$. 1D DCT is applied on the obtained feature vector. As per as DCT is concerned, according to energy compaction property, signal has a tendency that most of the energy portion lies in the low frequency components. Thus low frequency components of all samples generated after application of DCT are further explored for recognition purpose. Thus we can recognize an unknown numeral by finding the prototype numeral whose DCT coefficients are best fitted for that unknown numeral. The accuracy of the method depends upon the number of coefficients selected after application of DCT. Experimental 


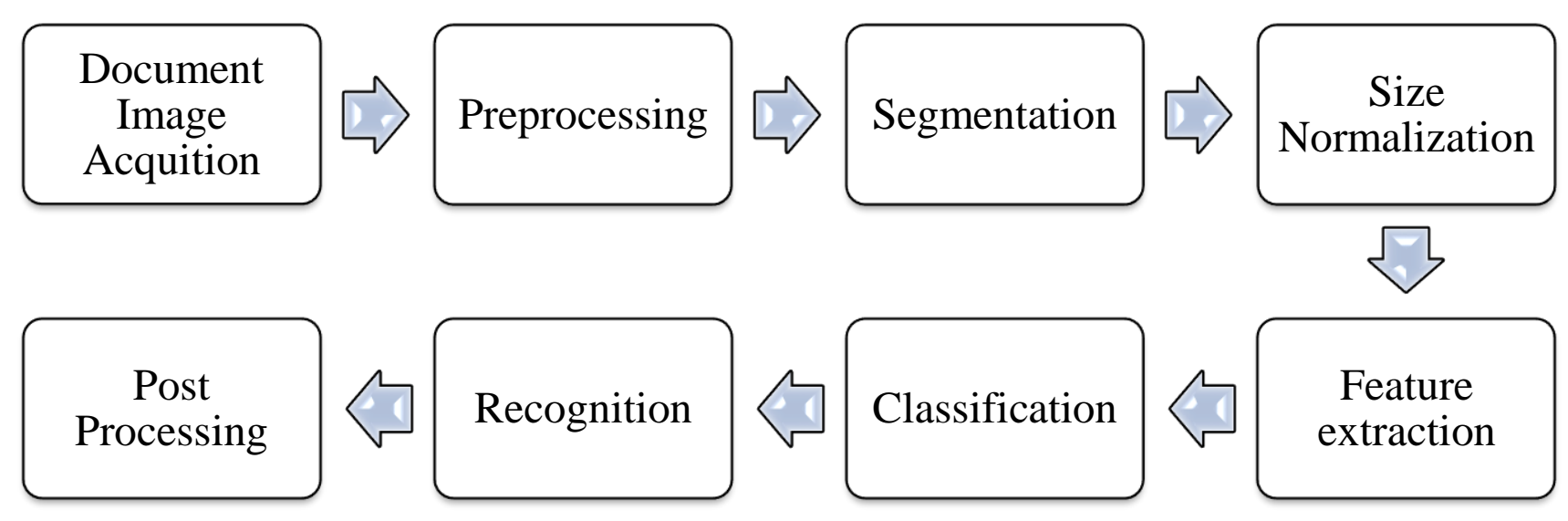

Figure.1 System architecture for numeral recognition

results show that our approach performs well in this application domain.

\section{Proposed approach for Devanagari numeral recognition}

In this paper, DCT based features are considered for Devanagari numeral recognition. Using the horizontal and vertical summation profile features along with diagonally scanned vectors are created. These vectors contain the major information about horizontal, vertical and diagonal lines used in the numeral image. Discrete Cosine Transform is applied on these four vectors to obtain new transformed values. These features are removed to determine cross-line features. Thus search can be reduced by selecting only few components from the transformed vector. Document image scanners connected to the machine is used to acquire the document images.

Image Enhancement techniques are used to get better quality image for enhanced features. Segmentation algorithm, bounding box method can be applied, so that interested part or block from the whole image can be separated to get required numeral. Segmented parts are then normalized to get consistency in the feature dataset in generalized format.

Database creation: Database required for experimentation is created at our institute by scanning the handwritten documents containing Marathi numerals. Documents are created with the help of school students studying at Zilha Parishad Primary School, Yelegaon village from district Nanded situated in Maharashtra state. The hardware used for scanning the documents is Samsung SCX3401 multifunction machine. The resolution of 600 dpi (dots per inch) is set for scanning the handwritten documents. The sample scanned page is shown in Fig. 2. Using horizontal and vertical projection profile method of segmentation, individual numbers are separated. These numerals are stored in folder for further processing. We have collected around 6000 sample numerals for the work. Every numeral from 0 to 9 are written same number of times, so the database created will have same number of samples with even distribution for each Marathi numeral. Segmented numbers are divided into a training set and testing set for experimentation.

\subsection{Preprocessing}

Pre-processing step in recognition includes noise removal, skew correction, slant removal and binarization. In Marathi numeral recognition, noise is mainly a very small pixel made by some dirt and writing style of individual. After receiving source document image, it has to be pre-processed. In this phase noise reduction and image enhancement algorithms applied to obtain a good quality image. Image is firstly converted into gray scale image and then to the binary format by using some threshold algorithm.

There may be some problems which are caused due to noise, illumination in image and other problems related to typing degradation. In some documents local thresholds can be calculated each line or word and based on the threshold related to that word, binarization can be done. In our data set the documents used are well scanned without any degradation in the ink used. The pre-processing techniques which are carried out in this work are explained below.

The OSTU [28] method is employed to convert the gray scale image into binary image. The advantage of using this method is that it chooses threshold automatically to reduce the intra class difference between black and white pixels. 


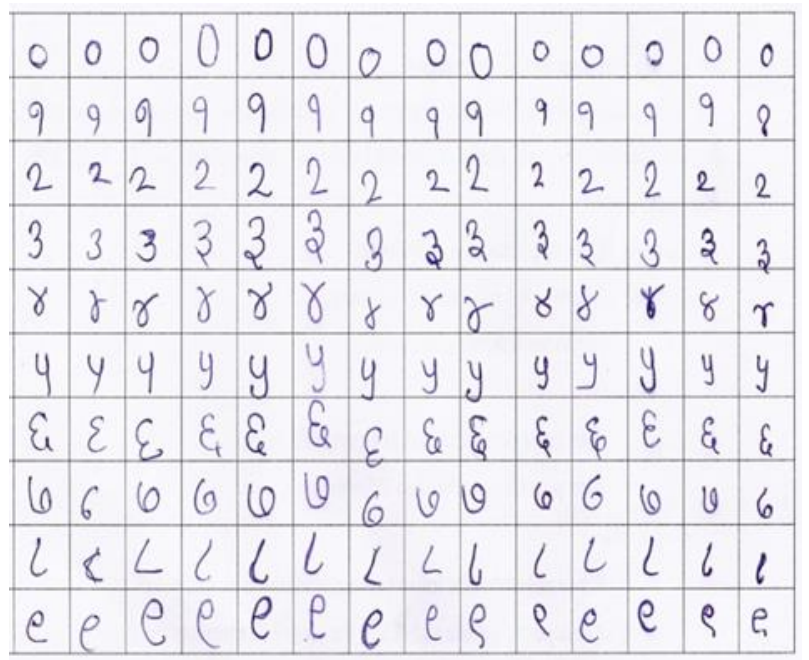

Figure.2 Sample document image

$$
\sigma_{w}{ }^{2}(t)=w_{0}(t) \sigma_{0}^{2}(t)+w_{1}(t) \sigma_{1}^{2}(t)
$$

$W_{0}$ and $W_{1}$ are the probabilities of the two classes separated by a threshold $t$ and $\sigma_{0}^{2}$ and $\sigma_{1}^{2}$ are the variances of these two classes. The class probability $W_{0}$ and $W_{1}$ is computed from the L histogram

$$
\begin{aligned}
& w_{0}(t)=\sum_{i=o}^{t-1} p(i) \\
& w_{1}(t)=\sum_{i=t}^{L-1} p(i)
\end{aligned}
$$

The edge and the bounding box of the image is computed. There are various ways of obtaining edge of image such as Robert, Sobel, Laplace and Canny. We have used Canny edge detection method in our system. This is because it can obtain background information more effectively.

\subsection{Segmentation}

Many algorithms to partition the required object from the given image are available. Internal segmentation and External segmentation are used by many researchers for creating individual sets of characters or numerals. Generally a skewed document segmentation is difficult using projection profile method. To get better segmented characters, skew correction algorithm is applied on the document. Hough transform is globally accepted algorithm for skew correction which gives comparable results is used for removing the skew from the documents. Thus corrected document image is processed further for segmentation.

Horizontal and vertical projection profiles are
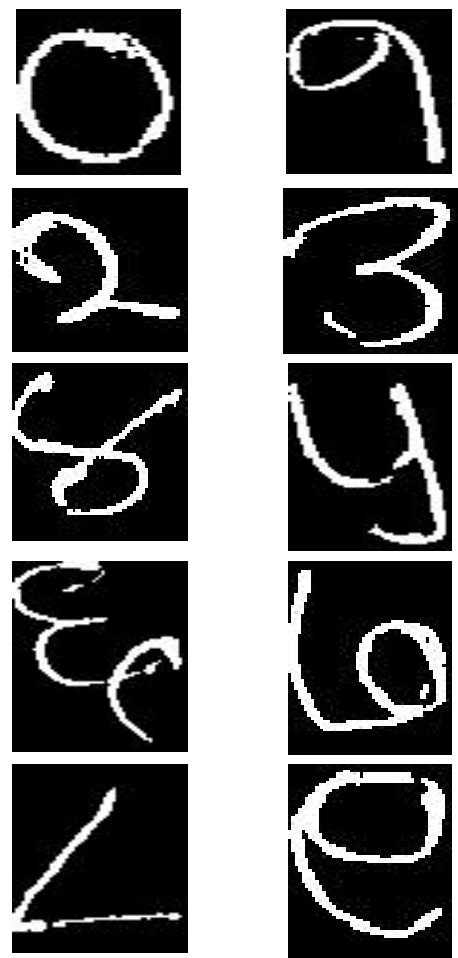

Figure.3 Segmented individual numerals

used to segment the individual numerals from the document. First horizontal projections are calculated to separate the horizontal lines from the document image. Based on the projection histogram minimum value is calculated to separate the lines. After getting the separated horizontal series of numbers, vertical projection profile is applied to separate the digits. Vertical projections are also scanned to find the zero or minimum value of histogram to separate the individual numerals to create the database as shown in Fig.3.

In the process of database storage, the individual sample points indicating numerals are cropped and inserted into database. For printed number, it is a simple task and requires no additional modifications as the size and orientation of the number is the same, but for other documents having handwritten numerals, for different person is having different writing style therefore it can be quite difficult. In addition, the person with the personal writing style to make radical changes in the normal partition is very cumbersome.

\subsection{Normalization}

These segmented numbers may vary in either shape or size. As the segmented image size changes, feature related to that number changes, which will lead to an unexpected result. To solve the problem, segmented characters are scaled to proper dimensions to get standard results in training as well 
as testing. Thus any size numeric identification is possible with minimal error. Normal size for the selected basic sample has overall greater impact on system performance. Size is bigger, the validation error is low, but time complexity has adverse effects and will be more. To get optimum performance, size of segmented numeral is normalized to $32 \times 32$ pixel.

\subsection{Feature vector generation}

1) Zonal Discrete Cosine Transform: In this approach by [31] numeral image is normalized to size of $64 \times 64$. This image is sub divided into blocks of size $8 \times 8$. After applying DCT on these blocks new transformed matrix is generated. Most of the information contents are concentrated towards the low frequency components present at the upper left corner of transformed matrix. As most of the energy is concentrated towards upper left corner, only few DCT coefficients were selected to form feature vector from each of the $8 \times 8$ blocks. In experimentation, 10 components from block are selected which leads to generation of feature set of 640 dimension for each numeral image. Numeral image is the sum of sinusoids of varying frequencies and magnitude represented by DCT coefficients. Most of the visually significant information may be concentrated in just a few coefficients of the DCT. Out of 640 dimensions 320 features i.e 5 features from each sub block are selected for future classification. Accuracy computed for this method is found 83.56.

2) Discrete Wavelet Transform: $2 D$ DWT is used to compute the features of numeral images [33]. Numeral image is decomposed into four different components using a low-pass filter (LPF) and a high-pass filter (HPF) by using DWT. A LPF and HPF are applied to each row/column of the image to get one low-frequency sub-band (LL) and three high frequency sub-bands (LH, HL, HH). The LL represents the horizontal and vertical low frequencies. The $\mathrm{HH}, \mathrm{HL}$ and $\mathrm{LH}$ and are termed as the diagonal, horizontal sub-band and vertical subband coefficients respectively. HL represents vertical low and horizontal high frequency. LH represents the vertical high and horizontal low frequency. Similarly, the HH represents vertical high and horizontal frequency components. Haar wavelet is used to decompose each numeral image into three levels. Most of the energy is concentrated towards the low frequency components in DWT which gives closer approximation to the original image. These details are being used for feature

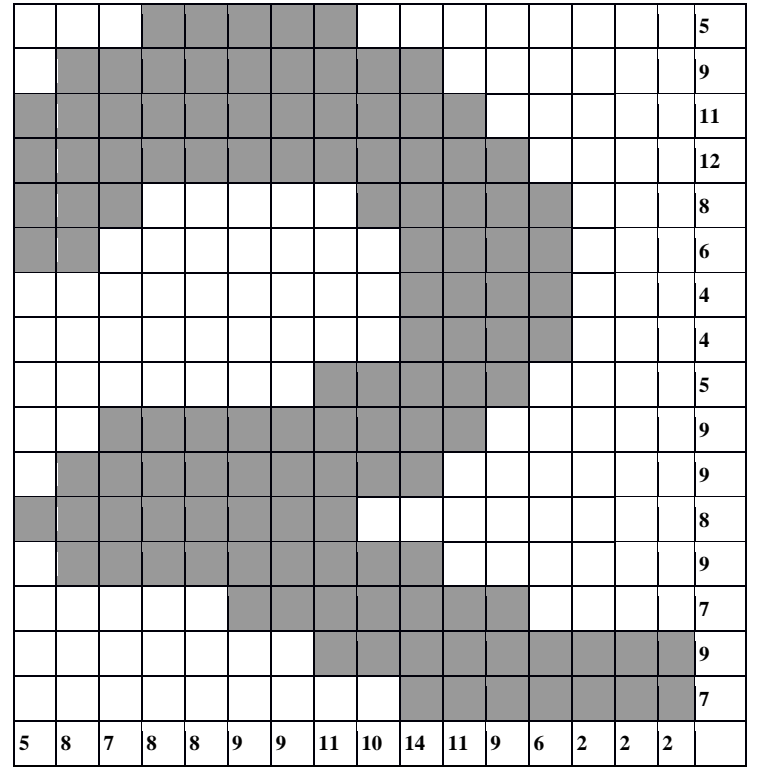

Figure.4 Summation vector generation

vector generation. After the application of Haar transform on the numeral image of size $128 \times 128$, the horizontal, vertical and diagonal details are obtained to get matrices of size $64 \times 64$ respectively. These are down sampled to get the final feature vector of size $1 \times 6144$. These components are selected as feature vector for classification.

3) Proposed 1D Discrete Cosine Transform on projection profiles: The binary numeral image with normal size of $32 \times 32$ is used for generating summation vectors in horizontal, vertical and diagonal directions Fig. 4 shows the binary pattern of numeral 2.

$$
\begin{aligned}
& H(j)=\sum_{i=1}^{n} I(i, j) \forall j=1,2,3 \ldots \ldots n \\
& V(i)=\sum_{j=1}^{n} I(i, j) \forall i=1,2,3 \ldots . n \\
& D(i)=\sum_{j=1}^{n} I(i, j) \forall i=1,2,3 \ldots . . j \\
& D^{\prime}(j)=\sum_{i=1}^{n} I(j, i) \forall j=1,2,3 \ldots . . i
\end{aligned}
$$

Pixels in horizontal vector, vertical vector and diagonally scanned vectors are calculated. These vectors obtained $H(j), V(i), D(i)$ and $D^{\prime}(j)$ are used for further processing.

1D DCT is applied on these four vectors to get transformed vector. Thus only first twenty five percent of the transformed components from each vector are considered for creation of feature vector. These all four vectors are then concatenated which leads to generation of feature vector containing 24 different DCT components.

Histogram of gradients (HOG) vector is calculated for the normalized numeral image. After getting this vector 1D DCT is applied to get transformed coefficients for the same vector. Thus 
only first 25 percent of the coefficients from the vector are selected. These features are concatenated with previous 24 features to form a new feature vector. Based on these features, numerals are compared for classification. In this paper only forward 1D DCT is used to extract the features vector using energy compaction property of DCT. In most of the research work inverse DCT is used for further processing but in the presented work only forward DCT is used to create feature vector. The equation for 1D DCT [22] is defined by following equation

$$
\begin{array}{r}
F(u)=\left(\frac{2}{N}\right)^{\frac{1}{2}} \sum_{I=0}^{N-1} A(i) \cdot \cos \left[\frac{\pi \cdot u}{2 \cdot N}(2 i+1)\right] f(i) \\
\text { where, } \quad A(i)= \begin{cases}\frac{1}{\sqrt{2}} & \text { for } \xi=0 \\
1 & \text { otherwise }\end{cases}
\end{array}
$$

4) Artificial Neural Network: Neural systems which are pretty much heuristic in nature are to a great extension reasonable for this sort of problems of recognition. Different types of neural network systems are implemented for OCR classification. A neural system is a registering design that comprises of enormously parallel interconnection of versatile neural processors. Neural system designs can be named, feed forward and back propagation systems. The most well-known neural systems utilized as a part of the OCR frameworks are the multilayer perceptron (MLP) of the Feed forward systems and the Kohonen's Self Organizing Maps (SOM).

We utilized a feed forward neural network system that maps sets of features to recognize the samples. This system is utilized with three layers including input layer, output layer and hidden layers for two unique sorts of element set. Output of one layer neuron is fed forward to the next layer. Every hidden layer has corresponding weight w0 to be adjusted. In ANN, every node in the system is neuron with a nonlinear actuation function. In this procedure supervised learning is utilized. Input layer is used to feed the raw data without any processing. Every hidden node determines the weighted sum of corresponding inputs and manipulates the output of the node by using threshold function which can be either a step function or a sigmoid function. Output node are computed based on type of problem given to neural network.

In the classification of numerals, the feature vector generated is fed to the neural network. The neural network consists of ten nodes in the output layer representing ten classes of ten numerals. In training phase, the weights are adjusted to get required class output for the numeral. Thus trained neural network is used for testing purpose. In the experimentation half of the numerals are used for training and remaining half numeral database is used for testing or recognition purpose.

\section{Experimental results}

Training set of the first fifty percent normalized samples in a data set is used for classification using artificial neural network. In the training weights are adjusted to get specified output. Thus using this data and trained ANN, the numeral features from testing data are fed to the ANN.

The accuracy of the network is calculated based on correctly classified samples. By changing the samples in training and testing data set, the accuracy is calculated. Artificial Neural Network is trained by using 50:50 (training : testing) samples in pass one. Then the ratio of 60:40, 70:30 and 80:20 are selected. Based on the ratio of number of samples in the train set to test set, the accuracy varies. If the ratio is having larger value, accuracy will be more. Classification rate of individual Marathi numeral from zero to nine is calculated the using ANN for all samples. After experimentation it is found minimum accuracy is found for numeral 3 and the maximum accuracy is investigated for numeral 4 .

Table 2 illustrates the recognition rate investigated during testing using fifty percent of numerals based on selected features. Thus the average classification rate of $90.30 \%$ is observed on the test set. As per as time complexity is concerned, 25 percent features are selected in final stage for classification and recognition. The number of comparisons required for finding nearest match depends upon number of features. If the selected number of features is less, then number of comparisons is also less. For large dataset having 30000 samples, it will have great impact on time complexity. This gain in the time complexity is due to DCT application.

\section{Comparison with existing approaches}

Table 3 shows the comparison of accuracy of proposed approach with existing transform based approaches. JPEG algorithm for image compression is used by [29]. All steps for JPEG are used including quantization which requires large computational time for extracting the feature vector. It is obvious that larger the resolution of image greater will be the details. By using this property [30] proposed better accuracy for same JPEG based approach. After application of 2D DCT on input 
Table 3. Correctly classified Numerals using 1D DCT features

\begin{tabular}{cccc}
\hline \hline Numeral & $\begin{array}{l}\text { No. of samples } \\
\text { used for Testing }\end{array}$ & $\begin{array}{l}\text { Correctly } \\
\text { classified samples }\end{array}$ & \multicolumn{2}{l}{$\begin{array}{l}\text { Recognition } \\
\text { Rate }\end{array}$} \\
\hline 1 & 3000 & 2654 & 88.47 \\
\hline 2 & 3000 & 2549 & 84.94 \\
\hline 3 & 3000 & 2489 & 82.91 \\
\hline 4 & 3000 & 2911 & 96.94 \\
\hline 5 & 3000 & 2749 & 91.51 \\
\hline 6 & 3000 & 2694 & 89.65 \\
\hline 7 & 3000 & 2637 & 87.72 \\
\hline 8 & 3000 & 2847 & 94.68 \\
\hline 9 & 3000 & 2784 & 92.55 \\
\hline 0 & 3000 & 2817 & 93.62 \\
\hline
\end{tabular}

Table 2. Comparison with existing methods

\begin{tabular}{cccccc}
\hline \hline $\begin{array}{c}\text { Sr } \\
\text { No }\end{array}$ & Published By & Methodology & $\begin{array}{c}\text { image } \\
\text { size }\end{array}$ & Classifier & $\begin{array}{c}\text { Accuracy } \\
\%\end{array}$ \\
\hline 1 & Abdurazzag[29] & JPEG DCT & $40 \times 40$ & Euclidean distance & 52.00 \\
\hline 2 & Biradar, Subhedar[30] & JPEG DCT & $60 \times 60$ & Euclidean distance & 55.00 \\
\hline 3 & G Shiva Reddy[31] & ZDCT & $64 \times 64$ & Euclidean distances, log likelihoods & 83.56 \\
\hline 4 & T.K Mishra[32] & DCT & $256 \times 256$ & BPNN & 84.60 \\
\hline 5 & Thin Nu Lwin [33] & DWT & $32 \times 32$ & ANN Back propagation & 71.64 \\
\hline 6 & Abhisek Sethy [34] & DCT & $20 \times 20$ & ANN, MSE & 80.40 \\
\hline 7 & Proposed approach & 1D DCT & $32 \times 32$ & ANN & 90.30 \\
\hline
\end{tabular}

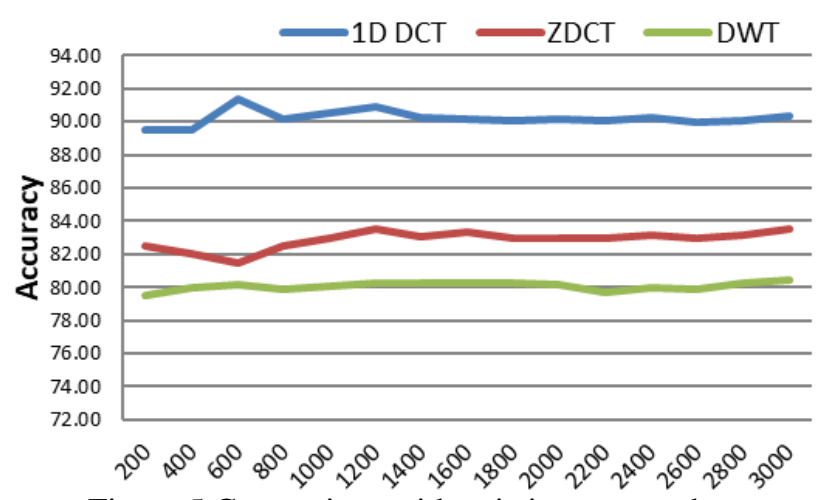

Figure.5 Comparison with existing approaches

image, Zonal DCT coefficients are selected for classification [31]. The size of input image [32] used is also very large as compared to our proposed method. DWT is also used for feature extraction but the accuracy found using this transform is not comparable. Abhishek Shethy [34] used very small size of input image as $20 \times 20$ but the accuracy obtained is for the method is very less.

Fig. 5 shows the comparative results based on best three approaches having optimum numeral image size. The samples are divided into equal number of intervals and selected to calculate accuracy using these techniques.

\section{Conclusions}

In the proposed approach, the features of the numeral data is calculate based on 1D DCT transform. Only forward DCT is used as data restoration is not required using inverse DCT (IDCT). Based on energy compaction property of DCT very few components having more information are selected for feature vector generation which reduces the number of samples in the vector in turn the number of comparisons required are also 
reduced. The features that received better recognition rate are used in recognition process to reduce the amount of time. The overall complexity of the system is the sum of complexity required for recognition as well as the time required to calculate the features. The recognition rate depends upon number of components used to generate feature vector. Average classification rate for the proposed approach is found to be $90.30 \%$ on given samples in the test dataset.

In future other feature extraction techniques can be applied for achieving better accuracy. Also more number of features lead to better result, so two or more approaches can be combined for further exploration. The validation process also plays very important role as better segmentation methods can be employed to separate the numerals without any overlap or without any noise. The paper discusses the system which is developed for dataset of Marathi handwritten numerals only. In future it can be extended to Devanagari characters or conjugate character recognition. In this approach database is created and then offline recognition is done. Thus the development can be further extended to the online recognition system.

\section{References}

[1] S.N. Srihari, "Recognition of handwritten and machine-printed text for postal address interpretation", Pattern Recognition Letters, Vol. 14, No. 4 pp. 291-302, 1993.

[2] W. Ying, Y. Lu, and P.F. Shi, "Handwritten Bangla numeral recognition system and its application to postal automation", Pattern Recognition, Vol. 40, No. 1, pp. 99-107, 2007.

[3] U. Pal and B. Chaudhari, "Indian Script Character Recognition: a Survey", Pattern Recognition, Vol. 37, pp. 1887-1899, 2004.

[4] R. Jayadevan, S. R. Kolhe, P. M. Patil, and U. Pal, "Offline recognition of Devanagari script: A survey", IEEE Transactions on Systems, Man, and Cybernetics, Part C (Applications and Reviews), Vol. 41, No. 6, pp.782-796, 2011.

[5] N. Das, J. M. Reddy, R. Sarkar, S. Basu, M. Kundu, M. Nasipuri, and D. K. Basu, "A statistical-topological feature combination for recognition of handwritten numerals", Journal on Applied Soft Computing, Vol. 12, pp. 24862495, 2012.

[6] M. Vaidya and Y. Joshi, "Marathi Numeral Recognition using statistical distribution features", In: Proc. of the IEEE conference on Information processing, pp. 586-591, 2015.
[7] M. Vaidya and Y. Joshi, "Handwritten Numeral Identification System Using Pixel Level Distribution Features", In: Proc. of the 2nd International Conference on Information and Communication Technology for Intelligent Systems, pp.1-9, 2017.

[8] U. Pal and B. B. Chaudhuri, "Automatic recognition of unconstrained off-line Bangla handwritten numerals", In: Proc. of the International conference on Advances in Multimodal Interfaces, Springer, Berlin, Heidelberg, pp. 371-378, 2000.

[9] K. Roy, T. Pal, U. Pal, and F. Kimura, "Oriya handwritten numeral recognition system", In: Proc. of the IEEE conference on Document Analysis and Recognition, pp. 770-774, 2005.

[10] G. Rajput and M. Hangarge, "Recognition of isolated handwritten Kannada numerals based on image fusion method", Pattern Recognition and Machine Intelligence, pp. 153-160, 2007.

[11]Z. Fan and R. Queiroz, "Maximum likelihood estimation of JPEG quantization table in the identification of bitmap compression history", In: Proc. of the IEEE International Conference on of Image Processing, pp. 948-951, 2000.

[12] N. Ahmed, T. Natarajan, and K. R. Rao, "Discrete cosine transform", IEEE Transactions on Computers, Vol. 100, No. 1, pp. 90-93, 1974.

[13] R. Plamondon and S. N. Srihari, "On-line and off-line hand-writing recognition: a comprehensive survey", IEEE Transactions Pattern Analysis \& Machine Intelligence, Vol. 22, No.1, pp. 63-84, 2000.

[14] U. Bhattacharya and B.B. Chaudhuri, "Handwritten Numeral Databases of Indian Scripts and Multistage Recognition of Mixed Numerals", IEEE Transaction on Pattern recognition and Machine Intelligence, Vol. 31, No. 3, pp. 444- 457, 2009.

[15] U. Garain and B. B. Chaudhuri, "Segmentation of Touching Characters in Printed Devnagari and Bangla Scripts Using Fuzzy Multifactorial Analysis", IEEE Transactions on Systems, Man, and Cybernetics-Part C Applications and Reviews, Vol. 32, No. 4, pp. 449-459, 2002.

[16] J. Han, M. Kamber, and J. Pei, Data Mining: Concepts and Techniques, Vol. 3, Morgan Kaufmann Publishers, July 2011.

[17] B. Shaw, S. K. Parui, and M. Shridhar, "Offline handwritten Devanagari word recognition: A segmentation based approach", In: Proc. of the International Conference on Pattern Recognition, Tampa, Florida, USA, pp. 1-4, 2008. 
[18] Z. Lu, Z. Chi, and W. Siu, "Extraction and optimization of B-spline PBD templates for recognition of connected handwritten digit strings", IEEE Transactions on Pattern Analysis and Machine Intelligence, Vol.24, No.1, pp.132-139, 2002.

[19] R. Buse, Z. Liu, and J. Bezdek, "Word recognition using fuzzy logic", IEEE Transactions on Fuzzy Systems, Vol.10, No.1, pp.65-76, 2002.

[20] J. Park, "An adaptive approach to offline handwritten word recognition", IEEE Transactions on Pattern Analysis and Machine Intelligence, Vol.24, No.7, pp.920-931, 2002.

[21] Y. Tan, W. Huang, Z. Yu, and Y. Xu, "Imaged document text retrieval without OCR", IEEE Transactions on Pattern Analysis and Machine Intelligence, Vol.24, No.6, pp. 838-844, 2002.

[22] M. Cannon, M. Fugate, D. R. Hush, and C. Scove, "Selecting a restoration technique to minimize OCR error", IEEE Transactions on Neural Networks, Vol.14, No.3, pp. 478-490, 2003.

[23] D. Shi, S.R. Gunn, and R.I. Damper, "Handwritten Chinese radical recognition using nonlinear active shape models", IEEE Transactions on Pattern Analysis and Machine Intelligence, Vol.25, No.2, pp. 277- 280, 2003.

[24] L.S. Oliveira, R. Sabourin, F. Bortolozzi, and C.Y. Suen, "Automatic recognition of handwritten numerical strings: a recognition and verification strategy", IEEE Transactions on Pattern Analysis and Machine Intelligence, Vol. 24, No.11, pp.1438-1454, 2002.

[25] A. R. Chadha, P. P. Vaidya, and M.M. Roja, "Face recognition using discrete cosine transform for global and local features", In: Proc. of the IEEE International Conference on of Recent Advancements in Electrical, Electronics and Control Engineering, pp. 502505, 2011.

[26] A. Thamizharasi and J. Jayasudha, "An Illumination Invariant Face Recognition by Selection of DCT Coefficients", International Journal of Image Processing, Vol. 10, issue 01, pp. 14-21, 2016.

[27] S.R. Saidas, T. Rohithram, K. Sanoj, and M. Manuel, "Malayalam Character Recognition using Discrete Cosine Transform", International Journal of Engineering and Computer Science, Vol. 5, No. 2, pp. 1574115743, 2016.

[28] N. Otsu, "A threshold selection method from gray-level histograms", IEEE Transactions on
Systems, Man, and Cybernetics, Vol. 9, No. 1, pp. 62-66, 1979.

[29] A. Aburas and S.A. Rehiel, "JPEG for Arabic Handwritten Character Recognition: Add a Dimension of Application", In Advances in Robotics, Automation and Control, InTech, pp. 21-32, 2008.

[30] G. Birajdar and M. Subhedar, "Use of JPEG Algorithm In Handwritten Devnagri Numeral Recognition", International Journal of Distributed and Parallel Systems, Vol.2, No.4, pp. 152-160, 2011.

[31] G. Reddy, P. Sharma, S. R. M. Prasanna, C. Mahanta, and L. Sharma, "Combined online and offline assamese handwritten numeral recognizer", In: Proc. of the IEEE National Conference on Communications, pp. 1-5, 2012.

[32] T. Mishra, B. Majhi, and S. Panda, "A comparative analysis of image transformations for handwritten Odia numeral recognition", In: Proc. of the IEEE International Conference on Advances in Computing, Communications and Informatics, pp. 790-793, 2013.

[33] T. Lwin and T. Soe, "Comparison of Handwriting characters Accuracy using Different Feature Extraction Methods", International Journal of Scientific Engineering and Technology Research, Vol. 3, No. 6, pp. 1027-1032, 2014.

[34] A. Sethy and P. K. Patra, "Off-line Odia handwritten numeral recognition using neural network: A comparative analysis", In: Proc. of the IEEE International Conference on Computing, Communication and Automation, pp.1099-1103, 2016. 